\title{
Pityriasis Rosea during Etanercept Treatment for Rheumatoid Arthritis Associated with Primary Biliary Cirrhosis
}

\author{
Oulkadi Lamia $^{1 *}$, AMINE Bouchra ${ }^{1}$, Elbinoune Imane ${ }^{1}$, Rostom Samira ${ }^{1}$, Bahiri Rachid ${ }^{1}$ \\ ${ }^{1}$ Department of Rheumatology A, El Ayachi Hospital, Ibn Sina University Hospital, Salé - Morocco
}

DOI: $10.36348 /$ sjpm.2021.v06i02.004

| Received: 25.01.2021 | Accepted: 06.02.2021 | Published: 12.02.2021

*Corresponding author: Doctor OULKADI Lamia

\section{Abstract}

In the pathogenesis of rheumatoid arthritis and primary biliary cirrhosis, the tumor necrosis factor-alpha has an important role. We describe the case of a 61-years-old woman with rheumatoid arthritis and primary biliary cirrhosis treated with anti-tumor necrosis factor-alpha agents. During treatment with golimumab, we found an improvement in liver function, but rheumatoid arthritis remained poorly controlled. When etanercept was started, the disease activity of rheumatoid arthritis was significantly improved and liver function was also improved despite the appearance of Pityriasis Rosea during etanercept. This case shows that etanercept therapy maintained liver enzymes within normal limits in PBC and controlled arthritis with a 10-month follow-up but the maintenance of this treatment was limited by the appearance of the pityriasis rosea.

Keywords: Rheumatoid arthritis; Primary biliary cirrhosis; TNF alpha antagonists, Etanercept, pityriasis rosea.

Copyright () 2021 The Author(s): This is an open-access article distributed under the terms of the Creative Commons Attribution 4.0 International License (CC BY-NC 4.0) which permits unrestricted use, distribution, and reproduction in any medium for non-commercial use provided the original author and source are credited.

\section{INTRODUCTION}

Primary biliary cirrhosis (PBC) is a chronic autoimmune hepatopathy characterized by the progressive destruction of the small intrahepatic bile ducts, causing fibrosis and possible cirrhosis [1]. The development of the disease is associated with the production of characteristic autoantibodies, including antimitochondrial antibodies, detected at titers equal to or greater than $1 / 40$ [2]. PBC is often found in the asymptomatic phase when only serum liver function tests are high and it may be associated with other autoimmune diseases, such as rheumatoid arthritis (RA) $[1,3]$. RA is a chronic autoimmune disease in which tumor necrosis factor-alpha (TNF $\alpha$ ) has an important pathogenic role as well as in PBC [4]. Etanercept is a $\mathrm{TNF} \alpha$ inhibitor which is a fusion protein produced by recombinant DNA against TNF $\alpha$ and has been established as a treatment of RA [5] and can be effective in PBC [2]. On the other hand, all TNF inhibitors can cause side effects such as infections, neoplasms, and an autoimmune disease. Skin eruptions like pityriasis rosea have been also described during treatment by etanercept [6]. We will describe the effect of etanercept treatment in a patient with RA and PBC, and we present the first case of pityriasis rosea occurring during etanercept treatment for RA associated with PBC to our knowledge.

\section{CASE REPORT}

A 61-years-old woman has been diagnosed with RA since 2003, according to the criteria of the American College of Rheumatology. Despite the uncontrolled status of RA, the patient was lost to follow-up and did not attend programmed appointments with rheumatologists and she managed her symptoms with NSAIDs medications and prednisone for 5 years.

Since 2008, she has taken antirheumatic drugs; methotrexate (20 mg/week), and prednisone (7.5 $\mathrm{mg}$ /day) for nine years, with a satisfactory clinical and laboratory response. In September 2017, elevated liver enzymes alkaline phosphatase ALP (1200 UI/l; normal $40-500 \mathrm{UI} / \mathrm{l})$, and gamma-glutamyltransferase, GGT (120 UI/l; normal, 9- $36 \mathrm{UI} / \mathrm{l}$ ) were observed without hepatic cytolysis. No other abnormalities of hepatic function have been observed. The serologic evaluation of hepatitis A, B, C, E was negative and the abdominal ultrasound was normal, the anti-mitochondrial antibodies (AMAs) were negative. Hepatic biopsy samples showed non-suppressive destructive cholangitis characterized by mononuclear inflammatory cells surrounding a small bile duct and classified as 
stage I according to Ludwig's classification. She was treated with $400 \mathrm{mg} /$ day of ursodeoxycholic acid which improved liver tests.

In November 2017, MTX had to be stopped due to severe stomach pain, nausea, and vomiting. The patient maintained a high disease activity (Disease Activity Score DAS28 5.34). Therefore, we considered a biological treatment. There were no hepatological or other contraindications. Rituximab (two doses of 1,000 mg) has been instituted. In March 2018, four months after rituximab, the patient was admitted to our rheumatology department because of an unsatisfactory joint response. Laboratory tests revealed erythrocyte sedimentation rate ESR $(48 \mathrm{~mm} / 1 \mathrm{~h})$ and $\mathrm{C}$ reactive protein CRP (49 mg / L) were increased and abnormal hepatic function tests: ALP $800 \mathrm{U} / \mathrm{L}$ (normal 40-500) and GGT values $128 \mathrm{U} / \mathrm{L}$ (normal 9-36), alanine aminotransferase (ALT) $18 \mathrm{U} / \mathrm{L}$ (normal0-55), aspartate aminotransferase (AST) $20 \mathrm{U} / \mathrm{L}$ (normal 534). Due to the poor clinical response (DAS 28: 5.09) and persistent changes in liver function tests, treatment with Rituximab was discontinued, while treatment with ursodeoxycholic acid was continued. Thus, we decided to start Golimumab $50 \mathrm{mg}$ once a month subcutaneously. Four months later, in September 2018, hepatic function improved, but arthritis remained poorly controlled DAS28: 4.4 (ESR :60 mm / $1 \mathrm{~h}$ ) and CRP: $57 \mathrm{mg} / \mathrm{L}$ ).
In November 2018, treatment with Etanercept (25 mg ETA twice a week subcutaneously) was initiated. From the first 4 weeks, the patient experienced a significant improvement in her arthritic symptoms. As shown in Figure 1, RA disease activity improved significantly and hepatic function also improved after starting ETA.

In July 2019, nine months after the first injection of etanercept, the patient presented a rash-like erythematous round lesion covered with fine scales associated with pruritus along the trunk cleavage lines, with the configuration of a Christmas tree (Figure 2). The blood exams were normal including blood cell parameters, VDRL test and liver function tests. Human herpes virus 6 and/or 7 (HHV 6-7) DNA in the plasma has not been researched. The skin biopsy was realized showing an epidermal focal spongiosis and lymphocyte exocytosis with a superficial perivascular infiltrate of lymphocyte in the dermis. The diagnosis of Pityriasis rosea has been confirmed. The rashes disappear 6 weeks later without leaving a sequel under antihistamine when etanercept was stopped. Thus, in August 2019, Etanercept was reintroduced, and the same eruption appeared again. Then etanercept has been definitively stopped and PR disappears completely.

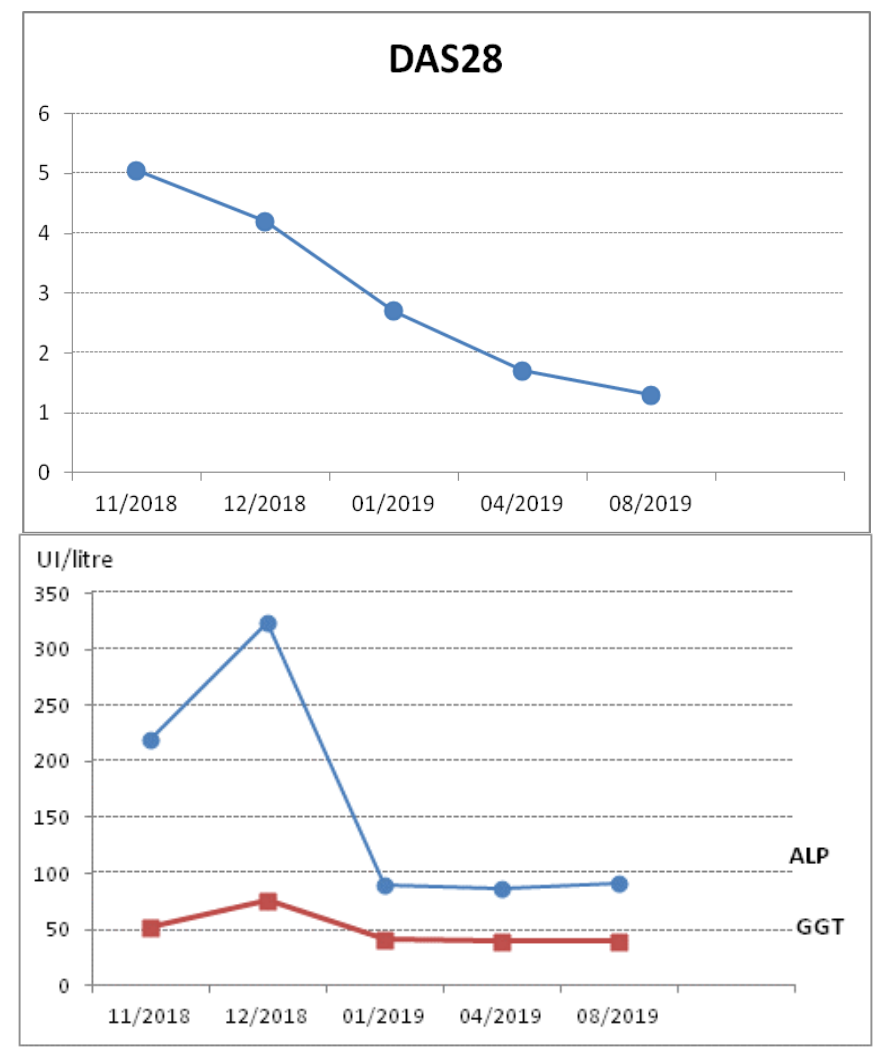

Fig-1: Changes in disease activity score (DAS28), ALP, and GGT over treatment with Etanercept. 


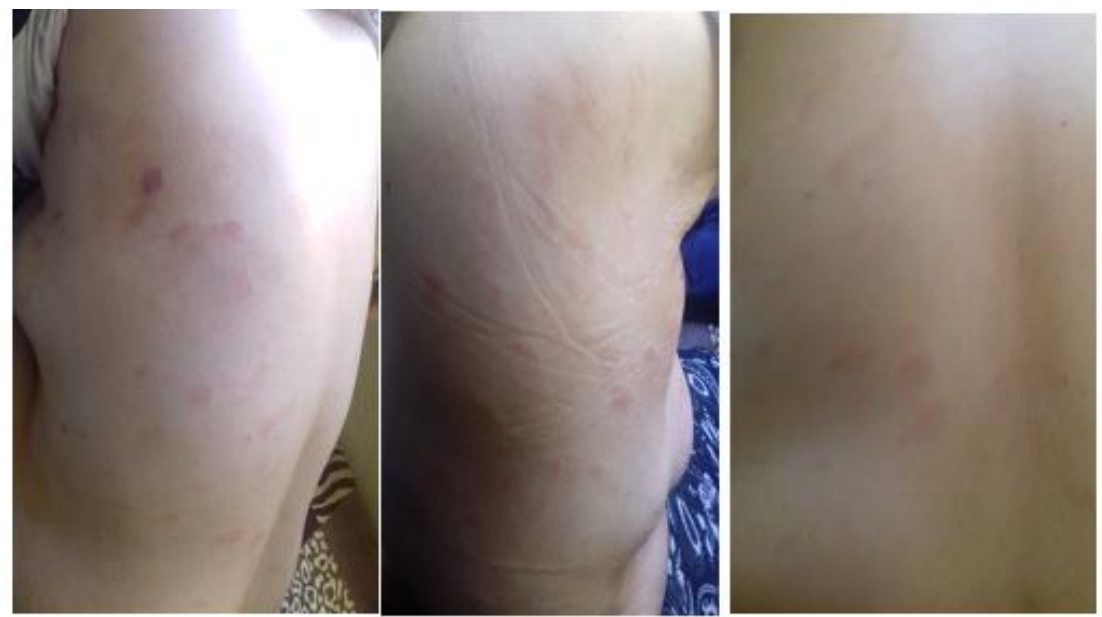

Fig-2: Eruption with erythematosquamous lesions following on the trunk.

\section{DISCUSSION}

We report the case of a patient with RA and $\mathrm{PBC}$ in whom Etanercept administration resulted in remission of RA and maintenance of a normal hepatic function, but the maintenance of this treatment was limited by the appearance of the pityriasis rosea.

PBC is a chronic autoimmune disease characterized by intrahepatic bile duct epithelial injury. It may be associated with other autoimmune conditions, including RA [7, 8]. Although $95 \%$ of patients with PBC are seropositive for antimitochondrial antibodies (AMAs) to the internal mitochondrial membrane, pyruvate dehydrogenase, and 2-oxoacid enzymes, AMA-negative cases do not differ clinically from those positive for AMA so the biopsy confirms the diagnostic. Besides, TNF $\alpha$ and associated genes are involved in the pathogenesis of PBC [3]. It was the most abundant cytokine, followed by TGF beta in bile duct epithelial cells in a group of patients with PBC [9]; TNF alpha mRNA levels in resting peripheral blood mononuclear cells was higher in patients with PBC than in normal controls or patients with other liver diseases [10]. Therefore, biologic treatments can be used primarily in RA-associated PBC when the underlying rheumatic condition is the primary indication for this treatment. The first case of RA-PBC with a successful response of the TNFa inhibitor was published by Spadaro et al. [2] in 2008. Due to the high activity of the disease, infliximab was administered initially but had little effect on RA activity and liver function test results. However, another case had suggested that infliximab which has been administrated as a therapy for RA, was well tolerated in the treatment of RA associated with PBC [11]. Etanercept treatment subsequently resulted in a significant reduction in activity in both diseases [2, 12, 13]. Attila Kovács et al. and Satoshi Kubo et al. have suggested a good efficacy and tolerability of this treatement[12,13]. Pityriasis rosea (PR) is an acute inflammatory dermatitis which can be persistent or recurrent. Its etiology has not been established, but a viral origin has been suspected for several years. Recently, the evidence suggests the role of the human herpesvirus (HHV) in the etiopathogenesis of PR [14, 15]. Reduced levels of natural killer cells and B cell activity in PR lesions were observed. This indicates the role of T-cell mediated immunity. Also, increased amounts of CD4 T cells and Langerhans cells have been found in the dermis, which may indicate antigen processing and presentation viral. However, this issue is still debated as some people are infected with HHV 6-7 and do not develop the disease. The diagnosis of PR is essentially clinical and a biopsy may be necessary. The appearance of PR under the TNF- $\alpha$ inhibitors can be explained by the role of this molecule in viral reactivation by lowering immunity. TNF- $\alpha$ plays a role in skin immunity defense mechanism, by its effects on inflammatory cells recruitment [16]. Therefore, the use of TNF- $\alpha$ inhibitors has been associated with an increased risk of infections. TNF- $\alpha$ inhibitors should be continued if they are not the signs related to PR like which are, lack of the herald patch, larger lesions, oral lesions, longer duration which are associated with the skin reaction to a drug [17]. Our case is the four reports to have developed the PR under Etanercept. There are only four cases reports describing PR during the administration of a TNF alpha inhibitor: one by adalimumab and three by etanercept, with good evolution of PR $[6,18,19]$.

In conclusion, Etanercept appear to be extremely effective in the treatment of RA and PBC. As our case and other case reports suggest. Our case puts the focus on the PR that might be encountered in clinical practice although it remains a rare side effect under TNF alpha inhibitor.

\section{ACKNOWLEDGEMENTS} all authors'

I am grateful for the help and the assistance of

Authors' contributions: LO drafted this manuscript, collected the data and reviewed the literature. BA, IE, SR and RB reviewed critically the manuscript. All authors have read and approved the final manuscript. 


\section{Funding}

There was no funding for the research concerning this manuscript.

\section{Consent for publication}

Written informed consent for publication was obtained from the patient.

\section{Competing interests}

The authors declare that they have no Competing interests

\section{REFERENCES}

1. Kaplan, M.M., Gershwin, M. (2005). Primary biliary cirrhosis. $N$ Engl J Med, 353:1261-73.

2. Spadaro, A., Scrivo, R., Riccieri, V., \& Valesini, G. (2008). Effect of tumor necrosis factor alpha antagonists in a patient with rheumatoid arthritis and primary biliary cirrhosis. Joint bone spine, 75(1), 87-89.

3. Gershwin, M. E., \& Mackay, I. R. (2008). The causes of primary biliary cirrhosis: convenient and inconvenient truths. Hepatology, 47(2), 737-745.

4. Purohit, T., \& Cappell, M. S. (2015). Primary biliary cirrhosis: Pathophysiology, clinical presentation and therapy. World journal of hepatology, 7(7), 926.

5. Yasoshima, M., Kono, N., Sugawara, H., Katayanagi, K., Harada, K., \& Nakanuma, Y. (1998). Increased expression of interleukin-6 and tumor necrosis factor-alpha in pathologic biliary epithelial cells: in situ and culture study. Laboratory investigation; a journal of technical methods and pathology, 78(1), 89-100.

6. Guarneri, C., Polimeni, G., Nunnari, G. (2009). Pityriasis rosea during etanercept therapy, European Review for Medical and Pharmacological Sciences, 13: 383-387

7. Neuberger, J. (1997). Primary biliary cirrhosis. Lancet, 350 (9081): 875-9.

8. Myasoedova, E., Davis, J. M., Crowson, C. S., \& Gabriel, S. E. (2010). Epidemiology of rheumatoid arthritis: rheumatoid arthritis and mortality. Current rheumatology reports, 12(5), 379-385.

9. Dienes, H. P., Lohse, A. W., Gerken, G., Schirmacher, P., Gallati, H., Löhr, H. F., \& zum Büschenfelde, K. M. (1997). Bile duct epithelia as target cells in primary biliary cirrhosis and primary sclerosing cholangitis. Virchows Archiv, 431(2), 119-124.

10. Larrea, E., \& Qian, C. (1994). Enhanced expression of $\mathrm{TNF} \alpha$ in patients with primary biliary cirrhosis. International Communications, 2(1), 6-13.

11. Dimopoulou, D., Dimitroulas, T., Akriviadis, E., \& Garyfallos, A. (2015). Infliximab as a treatment option for patients with rheumatoid arthritis and primary biliary cirrhosis. Rheumatology international, 35(11), 1913-1916.

12. Kovacs, A., Siminischi, A. G., Baksay, B., Gáll, A., Takacs, M., \& Szekanecz, Z. (2015). Successful etanercept treatment for primary biliary cirrhosis associated with rheumatoid arthritis. The Israel Medical Association journal: IMAJ, 17(2), 114-116.

13. Kubo, S., Iwata, S., Saito, K., \& Tanaka, Y. (2011). Successful treatment of primary biliary cirrhosis with etanercept in a patient with rheumatoid arthritis. Joint bone spine, 78(5), 535-536.

14. Drago, F., Malaguti, F., Ranieri, E., Losi, E., \& Rebora, A. (2002). Human herpes virus- like particles in pityriasis rosea lesions: an electron microscopy study. Journal of cutaneous pathology, 29(6), 359-361.

15. Broccolo, F., Drago, F., Careddu, A. M., Foglieni, C., Turbino, L., Cocuzza, C. E., ... \& Malnati, M. S. (2005). Additional evidence that pityriasis rosea is associated with reactivation of human herpesvirus-6 and-7. Journal of investigative dermatology, 124(6), 1234-1240.

16. Crum, N. F., Lederman, E. R., \& Wallace, M. R. (2005). Infections associated with tumor necrosis factor- $\alpha$ antagonists. Medicine, 84(5), 291-302.

17. Drago, F., Ciccarese, G., \& Parodi, A. (2018). Pityriasis rosea and pityriasis rosea-like eruptions: How to distinguish them?.JAAD case reports, 4(8), 800-801.

18. Errichetti, E., Piccirillo, A., Ricciuti, F., Ricciuti, F. (2011). Pityriasis rosea in a boy under treatment with etanercept. Esperienze Dermatologiche,13(4):179-81

19. Rajpara, S. M., Ormerod, A. D., \& Gallaway, L. (2007). Adalimumab- induced pityriasis rosea. Journal of the European Academy of Dermatology and Venereology, 21(9), 1294-1296. 